Bond University

Research Repository

\title{
Short-Term Training Cessation as a Method of Tapering to Improve Maximal Strength
}

Pritchard, Hayden J; Barnes, Matthew J; Stewart, Robin J C; Keogh, Justin W L; McGuigan, Michael R

Published in:

Journal of Strength and Conditioning Research

DOI:

10.1519/JSC.0000000000001803

Licence:

Other

Link to output in Bond University research repository.

Recommended citation(APA):

Pritchard, H. J., Barnes, M. J., Stewart, R. J. C., Keogh, J. W. L., \& McGuigan, M. R. (2018). Short-Term

Training Cessation as a Method of Tapering to Improve Maximal Strength. Journal of Strength and Conditioning Research, 32(2), 458-465. https://doi.org/10.1519/JSC.0000000000001803

\footnotetext{
General rights

Copyright and moral rights for the publications made accessible in the public portal are retained by the authors and/or other copyright owners and it is a condition of accessing publications that users recognise and abide by the legal requirements associated with these rights.
}

For more information, or if you believe that this document breaches copyright, please contact the Bond University research repository coordinator. 
Journal of Strength and Conditioning Research Publish Ahead of Print DOI: 10.1519/JSC.0000000000001803

Short term training cessation as a method of tapering to improve maximal strength

Training cessation as a method of tapering for strength

All research was conducted at the Human Performance Laboratory, UCOL, Palmerston North

Hayden J Pritchard, Matthew J Barnes, Robin JC Stewart, Justin WL Keogh \& Michael R McGuigan

Hayden J Pritchard - ${ }^{1}$ Lecturer in the Department of Exercise \& Sport Science, Faculty of Health Science at the Universal College of Learning, Palmerston North, New Zealand; and, ${ }^{2} \mathrm{PhD}$ student in the Department of Sport \& Recreation, Faculty of Health and Environmental Sciences, Auckland University of Technology, Auckland, New Zealand.

Dr Matthew J Barnes - Senior Lecturer in the School of Sport and Exercise at Massey University, Palmerston North, New Zealand.

Dr Robin JC Stewart - Lecturer in the Faculty of Health Science at the Universal College of Learning, Palmerston North, New Zealand.

A/Prof. Justin WL Keogh - Associate Professor in the: ${ }^{1}$ Faculty of Health Sciences and Medicine at Bond University, Queensland, Australia; ${ }^{2}$ Sports Performance Research Institute New Zealand, Auckland University of Technology, Auckland, New Zealand; and, ${ }^{3}$ Cluster for Health Improvement, Faculty of Science, Health, Education and Engineering, University of the Sunshine Coast, Queensland, Australia.

Prof. Michael R McGuigan - Professor at ${ }^{1}$ Sports Performance Research Institute New Zealand, Auckland University of Technology, Auckland, New Zealand; ${ }^{2}$ School of Medical and Health Sciences, Edith Cowan University, Perth, Australia.

Corresponding Author:

Hayden Pritchard

UCOL Exercise \& Sport Science

Private Bag 11022

Palmerston North 4442

New Zealand 
Phone: (06) 9527001 ext: 70666

Email: $\underline{\text { h.pritchard@ucol.ac.nz }}$

This manuscript is original and not previously published, nor is it being considered elsewhere until a decision is made as to its acceptability by the JSCR Editorial Review Board.

\section{ABSTRACT}

The aim of this study was to determine the effects of two different durations of training cessation on upper and lower body maximal strength performance, and to investigate the mechanisms underlying performance changes following short term training cessation. Eight resistance trained males $(23.8 \pm 5.4$ years, $79.6 \pm 10.2 \mathrm{~kg}, 1.80 \pm 0.06 \mathrm{~m}$, relative deadlift $1 \mathrm{RM}$ of $1.90 \pm 0.30$ times bodyweight) each completed two four-week strength training periods followed by either 3.5 days (3.68 \pm 0.12 days) or 5.5 days ( $5.71 \pm 0.13$ days) of training cessation. Testing occurred pre-training (T1), on the final day of training (T2) and following each respective period of training cessation (T3). Participants were tested for salivary testosterone and cortisol, plasma creatine kinase, psychological profiles, and performance tests (countermovement jump (CMJ), isometric mid-thigh pull (MTP) and isometric bench press (IBP)) on a force plate. Participants' bodyweight increased significantly over time $(P=0.022)$. CMJ height and IBP peak force showed significant increases over time $(P=0.013$, 0.048 and 0.004 , respectively). Post-hoc testing showing a significant increase between T1 and T3 for both CMJ height and IBP peak force $(P=0.022$ and 0.008 with effect sizes (ES) of 0.30 and 0.21 , respectively). No other significant differences were seen for any other measures. These results suggest that a short period of strength training cessation can have positive effects on maximal strength expression, perhaps due to decreases in neuromuscular fatigue.

Key words: peaking, performance, strength, tapering, training 


\section{INTRODUCTION}

Short-term training cessation is when an athlete ceases training for a short period of time whilst continuing with everyday activities (24). It has also been referred to as detraining, however because it can result in improved performance in the short term it does not conform strictly to the definition of detraining (22). Short term training cessation is sometimes undertaken as part of a taper (or as an alternate taper method) prior to an important event. Currently, little is known regarding the best duration of implementing training cessation during the taper. A better understanding of optimal durations, as well as understanding the mechanisms behind its effects, will assist exercise scientists and practitioners in effectively prescribing tapering practices for strength athletes.

Weiss et al. (31) investigated the effects of short term training cessation on exercise performance in 54 young male participants by training the seated heel raise movement three days per week for eight weeks. Participants showed significant improvements in isokinetic plantar flexor and one repetition maximum (1RM) seated heel raise strength following the training period. Participants were then split randomly into four groups which either ceased training for two, three, four or five days following the training period. The results showed that 1RM heel raise had small improvements at three $($ Effect size $(E S)=0.30)$ and four $(E S=0.38)$ days of training cessation in untrained participants, compared to only trivial improvements at other durations of training cessation. The four days of training cessation also had statistically significant improvements compared to two or five days of training cessation. Additionally, a small ES was also seen for isokinetic torque at 1.05 $\mathrm{rad} / \mathrm{s}$ following four days of training cessation $(E S=0.19)$ and a moderate $E S$ for isokinetic torque at $3.14 \mathrm{rad} / \mathrm{s}$ following four days of training cessation ( $E S=0.57$ ), with only trivial ES for other durations. 
A follow up study by Weiss et al. (32) had 25 strength trained males follow a periodized plan for four weeks, focused on heavy upper body resistance training. Participants were tested for their isokinetic bench press at moderately fast $(1.49 \mathrm{~m} / \mathrm{s})$ and slow $(0.37 \mathrm{~m} / \mathrm{s})$ velocities and 1RM bench press immediately following their four weeks of training and after two, three, four or five days of training cessation (depending on the group they assigned to). No significant changes were seen, however, maximal low velocity isokinetic force of the bench press showed small improvements in ES at four days of training cessation, with only trivial changes observed for other training cessation periods and tests. Anderson \& Cattanach (1) also found small improvements in 1RM bench press and squat strength when 41 track and field athletes had two to seven days off training following a five week, three days per week strength training program.

These results $(1,31,32)$ are somewhat consistent with the findings of Pritchard et al. (25) who reported that elite powerlifters usually take $3.7 \pm 1.6$ days off prior to important competitions. Together these studies suggest that short durations of training cessation can maintain, or perhaps even improve, strength performance. However, to date no studies have investigated the mechanisms behind the maintenance, or improvement, in performance observed during short term resistance training cessation, its effects on lower body muscle groups or used strength trained populations as participants.

Several studies have investigated longer durations of training cessation (greater than one week), where performance was more likely to be only maintained or reduced $(12,14)$. These studies typically also examined the anabolic hormonal and biochemical profile, noting reductions in markers of muscle damage and improved anabolic state during the cessation of training $(12,14)$. It has also been observed in longer duration training cessation studies that surface electromyography (EMG) and associated muscular performance decreases (non-significant) after two weeks of detraining (12). 
Therefore, improvements in performance during short duration training cessation may be related to improvements in neuromuscular activation and anabolic profile, which has yet to be studied. If training cessation results in improved neuromuscular activation or a more anabolic environment, this may result in improved strength performance due to greater motor unit activation or an increase in muscle mass and, thus, enhanced force output.

The aim of this study was to investigate the effects of different durations of training cessation on both upper and lower body maximal strength performance in resistance trained males. Additionally, the mechanisms underlying cessation related performance changes were explored. We hypothesized that training cessation will improve maximal strength, of both the upper and lower body, and that changes in hormonal profile, EMG and/or mood states will be associated with these positive changes in physical performance. It was also hypothesized that 3.5 days of training cessation would be more beneficial than 5.5 days.

\section{METHODS}

\section{Experimental Approach to the Problem}

This crossover trial study was designed to determine the performance effects and mechanisms underlying the effects of different durations of training cessation on maximal strength in resistance trained men. Specifically, participants were randomly assigned to either 3.5 days (84-hours condition A) or 5.5 days (132-hours - condition B) of training cessation following four-weeks of strength training. This ensured participants had a similar style of training prior to training cessation, ensuring the effects of the training cessation could be determined from a similar fatigue state. 
Participants were familiarized with all testing procedures at least 48-hours prior to any experimental testing. Participants reported to the lab fasted, at the same time ( $\mathbf{3 0} \mathbf{~} \mathbf{m i n})$, in the morning for testing prior to each four-week training period (T1), within 12 hours prior to each final training session (T2), and immediately following each training cessation period (T3). On each visit to the lab, participants provided a saliva sample, a finger-prick blood sample, completed two psychological surveys. Tests were performed to capture information related to physiological and psychological that may impact performance. Following these tests, performance tests (vertical jump, mid-thigh pull (MTP) and isometric bench press (IBP)) on a force plate. EMG was measured on MTP and IBP. These variables were chosen to determine the effects on maximal strength performance and neuromuscular function.

Following the first post-cessation testing, participants were given a 7-10 day "wash-out" period after which they completed another four-weeks of training followed by the other cessation intervention. The eight participants who completed the study did so in a counter-balanced fashion.

\section{Subjects}

Prior to commencing the research, the study was approved by the Auckland University of Technology Human Ethics Committee. All participants were informed of the benefits and risks of the investigation prior to providing written informed consent, before beginning any testing procedures. Inclusion criteria were: (i) participants current deadlift 1RM of at least 1.5 times bodyweight (BW), and (ii) two or more years of resistance training experience. 13 men, who met the inclusion criteria, volunteered and were recruited as participants within this study, however, due to various reasons, only eight completed the study. 
The eight participants who completed the study were aged $23.8 \pm 5.4$ years, had a body mass of 79.6 $\pm 10.2 \mathrm{~kg}$, a height of $1.80 \pm 0.06 \mathrm{~m}$ and a relative deadlift $1 \mathrm{RM}$ of $1.90 \pm 0.30$ times $\mathrm{BW}$ at the commencement of the study.

\section{Testing Procedures}

Strength Testing. Within one week of beginning the study, participants were tested for their 1RM on all powerlifts (squat, bench press and deadlift) according to NSCA guidelines (8). Participants were also tested for 2-8RM on all other lifts programmed (see program in table 1) to determine estimated $1 \mathrm{RM}$ on lifts that may be unfamiliar. Estimated $1 \mathrm{RM}$ was determined using the formula:

$$
\text { 1RM }=\text { Load } /(1.0278-0.0278 * \text { Reps Performed) }(2)
$$

1RM testing was repeated during the "wash-out" period in order to establish training loads for the subsequent, second four-week training period. This testing occurred at the same time of the day ( \pm 1 hour) on both occasions.

INSERT TABLE ONE ABOUT HERE

Endocrine and Biochemical Measures. Participants fasted from the previous evenings meal prior to testing sessions, this meal was noted and participants were instructed to replicate this meal the evening before each testing session throughout the study. Participants were instructed to avoid resistance exercise and minimize all exercise for $\mathbf{4 8}$ hours prior to each lab testing session (except for T2, as prescribed training was performed within this time frame), and to avoid all exercise for at least $\mathbf{2 4}$ hours prior to the testing session. All testing sessions occurred at the same time in the morning ( $\pm \mathbf{3 0} \mathrm{min}$ ) throughout the study. Water consumption was allowed ad-libitum prior to each testing session to ensure participants arrived hydrated. 
Participants were immediately seated upon arrival at the lab, given $250 \mathrm{ml}$ of water to drink and instructed to relax for five minutes. After this rest period, participants produced (through passive drooling) a saliva sample of at least $\mathbf{2} \mathbf{~ m L}$ into a $10 \mathrm{~mL}$ polypropylene collection tube (LabServ, Thermo Fisher, New Zealand). Salivary concentrations of testosterone and cortisol have been shown to be independent of flow rate (26). Samples were immediately separated into two polypropylene micro-centrifuge tubes (LabServ, Thermo Fisher, New Zealand) and placed on ice. Following the trial, samples were transferred to a freezer and stored at $-80^{\circ} \mathrm{C}$ until analysis. Analysis occurred in duplicate using commercial enzyme-linked immunosorbent assay (DRG International, USA) according to manufacturer's instructions. Coefficient of variation (CV) of duplicate samples was 3.3\% for cortisol and $5.3 \%$ for testosterone.

Following saliva collection, a capillary blood sample was collected from a finger using aseptic technique. This blood sample was immediately analyzed to determine plasma creatine kinase (CK) activity using the Reflotron ${ }^{\circledR}$ systems spectrophotometer (Roche Diagnostics, Switzerland) which has typical CV of 3.5\%. This method of CK analysis has been used previously in the literature to assess for indicators of muscle damage (13).

Psychological and perception measures. Participants completed a profile of mood states (POMS) (10) and daily analysis of life demands in athletes (DALDA) questionnaires (27). POMS was analyzed to determine total mood disturbance (TMD) score which represents all six specific mood states of the test. Previously, TMD scores have been reported to indicate athletes that may be at risk of staleness (or overtraining) and so this score may indicate whether there is any impact of the training or recovery on a participants psychological state (10). A baseline for DALDA (frequency of 'worse than normal' results) was established in the initial two weeks of training and compared to changes following the training and training cessation periods (27). The tool has been used previously with strength athletes to monitor responses to intense training (28). 
Performance measure: Participants performed a five-minute warm up at 70W on a cycle ergometer before performing normalization contractions for EMG, as outlined under 'Neuromuscular measures' section. Participants then performed practice CMJ's with hands on hips at $50 \%, 75 \%$ and $100 \%$ effort, followed by three recorded maximal CMJ's on a force plate (400 series, Fitness Technology, Australia) separated by approximately one-minute of rest. The force plate recorded at a rate of $600 \mathrm{~Hz}$ for all performance tests. The best (based on jump height) of the three CMJ's was then analyzed using commercial software (Ballistic Measurement Systems (BMS), Innervations, Australia) to determine jump height and flight-time: contraction time. These results were used to measure the effects of training cessation on maximal power and neuromuscular function $(3,29)$. CV's were $2.9 \%$ for CMJ height and 3.3\% for CMJ flight time: contraction time. Intra class correlations (ICC's) were 0.97 for $\mathrm{CMJ}$ height and 0.91 for $\mathrm{CMJ}$ flight time: contraction time.

The MTP was then performed on the same force-plate with the knees at $130^{\circ}$ (whereby a knee angle of $180^{\circ}$ represents full knee extension) and an upright torso using previously described methods $(19,20)$. Participants performed practice MTP's at $50 \%, 75 \%$ and $100 \%$ effort, followed by three recorded maximal MTP efforts of approximately five seconds on the force plate, separated by approximately one-minute of rest. Participants were instructed to pull as hard and fast as possible during each maximal effort. The best MTP (based on peak force) was analyzed to determine peak force and maximum rate of force development (mRFD). Peak force was the highest force recorded during the contraction, and mRFD was the maximum value calculated by dividing the change in force over the time interval. These values were automatically calculated using the BMS software for both MTP and IBP. MTP's have been used previously in the literature and have shown strong positive relationships with dynamic $1 \mathrm{RM}$ performance, particularly of the lower body $(19,20)$. CV's were 3.2\% for MTP peak force and $11.7 \%$ for MTP mRFD. ICC's were 0.97 for MTP peak force and 0.85 for MTP mRFD. 
The IBP was then performed on the same force plate with the top end of the bench centered over the force-plate and the elbows at $90^{\circ}$ flexion (if $180^{\circ}$ is straight), hands were positioned no more than $81 \mathrm{~cm}$ apart (15). Participants performed practice IBP's at 50\%, 75\% and 100\% effort, followed by three recorded maximal IBP efforts of approximately five seconds on the force plate, separated by one-minute of rest. Participants were instructed to push as hard and fast as possible during each maximal effort. The best IBP (based on peak force) was analyzed to determine peak force and mRFD as described for MTP. CV's were $4.6 \%$ for IBP peak force and $12.2 \%$ for IBP max mRFD. ICC's were 0.98 for IBP peak force and 0.88 for IBP mRFD.

Neuromuscular measures: Surface EMG of the vastus lateralis (VL) and triceps brachii (TB) were measured during MTP and IBP, respectively. The VL is a muscle that is easily accessible for EMG, and studies have shown that both throughout the entire deadlift and during the final $60^{\circ}$ of knee extension VL activation is similar to or higher than several hamstring muscles making it a suitable muscle to measure during the $\operatorname{MTP}(5,6)$. The TB is one of the agonist muscles during elbow extension and, as such, has a primary role during the bench press. Electrodes were placed according to the Surface Electromyography for the Non-Invasive Assessment of Muscles (SENIAM) recommendations (9).

EMG was measured using $10 \mathrm{~mm}$ diameter gel filled $\mathrm{Ag} / \mathrm{AgCl}$ electrodes (Blue Sensor, Medicostest, Rugmarken, Denmark) at a sampling rate of $1000 \mathrm{~Hz}$ via a PowerLab data acquisition system and analyzed in Chart for Windows (ADInstruments, Australia). The highest 1000ms average rectified EMG recorded during the greatest MTP or IBP was compared to the highest $1000 \mathrm{~ms}$ average rectified EMG recorded during maximal activation of the VL or TB, respectively. Normalization of VL EMG was determined using the highest $1000 \mathrm{~ms}$ average rectified EMG of three maximal five second isometric contractions of the quadriceps during a seated knee extension, with the knee angle set at 
$90^{\circ}$, and for the triceps during a standing elbow extension with the elbow angle at $90^{\circ}$. This maximal activation testing occurred immediately following the cycle warm up. The average rectified EMG during MTP and IBP was reported as a percentage of the average rectified EMG value obtained from the MVC and referred to as normalized EMG. These results may give an indication of the neural input to the musculature at differing time points during the study and may provide an indication of whether changes in force are due to alterations in neural activation and neuromuscular efficiency $(4,17)$.

\section{Training Protocol}

Following the first testing session participants commenced four-weeks of training (see table 1) focused on the powerlifts. The program aimed to bring all participants into a similar phase of training and fatigue prior to training cessation. This training was designed using Prilepin's chart as a guide to sets and repetitions at specific training intensities and the specific program used has been shown to be effective at improving maximal strength (23). The program consisted of four exercises per day; the first exercise was one of the three powerlifting competition lifts (back squat, bench press and deadlift), the next two exercises were usually a variation of the powerlifts, with the final exercise being an accessory movement aimed at producing muscular hypertrophy of some of the primary agonists. Participants completed a DALDA questionnaire prior to all training sessions, as well as recording their training sessions within a training log. A minimum of one full day's rest was instructed between training sessions, i.e. if day one was on Monday then day two would be on Wednesday at the earliest. During the training periods participants were able to continue performing aerobic and sport training as per normal (but no other resistance training), however this was discontinued during the training cessation periods and 48hrs prior to each initial testing session. During the 'wash-out' period between conditions participants could train as they wished. Training compliance was $97.8 \%$ over the two training periods. 


\section{Statistical Analysis}

Two-way repeated-measures ANOVA were used to test for differences in psychological (TMD and DALDA), performance (peak CMJ power, CMJ height, CMJ flight-time: contraction time, MTP and IBP peak force, and, MTP and IBP mRFD) and neuromuscular measures (normalized EMG), between time points and training cessation conditions. Where a significant difference was determined by ANOVA, post-hoc paired comparisons were made using the method of Student-Newman-Keuls. Significance was set at $\mathrm{P} \leq 0.05$. Analysis was performed using commercial computer software (Sigma Plot 11.0, Systat Software, Inc., Chicago, Illinois, USA).

ES of performance measures were calculated and interpreted as: trivial 0-0.2, small 0.2-0.6, moderate $0.6-1.2$, large 1.2-2.0 and very large $>2.0$ (11). CV's and ICC's for performance measures were calculated using the three maximal efforts recorded during T1 for the 3.5 days condition.

RESULTS

Due to participant availability for final training, the 3.5 day and 5.5 day training cessation periods were $3.68 \pm 0.12$ days ( $88.42 \pm 0.12$ hours) and $5.71 \pm 0.13$ days ( $136.94 \pm 0.13$ hours) of training cessation, respectively.

Participants bodyweights increased over time $(P=0.022)$, with post-hoc testing revealing a significant increase between $\mathrm{T} 1$ and $\mathrm{T} 2(0.85 \%, \mathrm{P}=0.036$, with a trivial ES of 0.05$)$ with no further change from T2 to T3. No difference in the changes in bodyweight were observed between conditions. Bodyweight changes are shown in Figure 1. 
INSERT FIGURE ONE ABOUT HERE

Table 2 shows the results of the performance tests. CMJ height showed significant increases over time $(P=0.013)$ with post-hoc testing showing a significant increase from $T 1$ to $T 3(P=0.022$, with a small ES of 0.30). MTP relative peak force approached a significant increase over time $(P=0.068)$ and no difference was found between training cessation durations $(P=0.682)$. IBP relative peak force showed significant increases over time $(P=0.004)$ with post-hoc testing revealing a significant increase from T1 to T3 $(P=0.011$, with a small ES of 0.30$)$. As with MTP, no difference between training cessation duration was found $(P=\mathbf{0 . 7 6 2})$. No other significant differences were seen between any of the conditions for any of the performance measures made.

INSERT TABLE TWO ABOUT HERE

No significant differences were seen at any time point during any condition for absolute or percentage changes in endocrine and biochemical measures (Table 3), DALDA 'worse than' scores, POMS TMD scores, or EMG results (Figures 2 and 3).

INSERT FIGURES TWO AND THREE ABOUT HERE

INSERT TABLE THREE ABOUT HERE 


\section{DISCUSSION}

The present results indicate that strength training followed by short term training cessation improved performance more than strength training alone. Significant performance improvements, compared to pre-training, were observed for both CMJ height and IBP relative peak force following short term training cessation, this significant improvement was not present on the final training day. No training cessation period was shown to be more beneficial, indicating that both 3.5 and 5.5 days off training have similar effects on performance.

Anderson \& Cattanach $(1)$ and Weiss at al. $(31,32)$ had previously demonstrated that short durations of training cessation, of less than a week, were able to enhance, or at least maintain, strength following a period of strength training. Of these studies, only Weiss et al. (31) showed statistically significant increases in maximal strength, and this was only assessed for the plantar flexors of the ankle. The present study is the first to demonstrate statistically significant increases in multi-joint, upper body strength following a period of training cessation, when compared to training alone. These findings indicate that short term training cessation, as a form of taper, may be effective for enhancing strength of the upper body and maintaining strength of the lower body and could be implemented within a training cycle.

The current investigation did not show either period of training cessation to be more beneficial than the other. Neither 3.5 nor 5.5 days off training demonstrated any significant differences for any performance measure, and ES's were of similar magnitudes regardless of condition. To date only one study (31) has shown statistically significant improvements in maximal strength for a specific time period, with four - compared with two and five - days of training cessation improving maximal strength of the plantar flexors, while having similar ES's to three days. A similar period of time off training ( $3.7 \pm 1.6$ days), prior to competition, has been reported by elite powerlifters $(25)$. Weiss et 
al. (32) showed small ES improvements at two and four days training cessation (compared with only trivial ES's for three and five days of training cessation). Similarly, Anderson \& Cattanach (1) did not show any period from two to seven days to be more optimal at improving maximal strength.

Therefore, it appears less than a week off training may be suitable for performance enhancements and/or maintenance with no particular time period more beneficial.

We observed that $\mathrm{CMJ}$ height increased following training cessation compared with pre-training values. As countermovement jumps are heavily reliant on the stretch-shortening cycle, efficient jumping performance may indicate that the neuromuscular system is functioning effectively (29). McLean et al. (21) and Twist et al. (30) have shown decreases in vertical jump performance (flight time) following competitive rugby league matches which was subsequently restored several days later. Twist et al. (30) attributed this decrease in performance to two factors: decreased central drive (due to muscle soreness) and tissue damage disrupting calcium release to impair excitation contraction coupling. Therefore, it could be hypothesized that more efficient neuromuscular function (or less neuromuscular fatigue) allowed for better expression of maximal strength in the current study and may underpin the potential performance gains associated with short term training cessation.

Several other physiological changes occurred which may assist in explaining the performance changes observed. One such change was that bodyweight showed statistically significant increases over the training period (albeit by a small amount). Increases in bodyweight may indicate muscle mass was gained during the training periods. Although muscle mass was not directly measured, increases are commonly seen following strength training (18). Improvements in muscle mass could cause improvements in strength following training due to an increased size of muscle fibers and thus force production capacity (7). It was also interesting to note that, although not significant, T3 had lower cortisol values in both conditions, suggesting less stress following the training cessation 
periods (16). As no other significant changes were found for other biochemical, hormonal or neuromuscular measures, further investigation may assist to confirm this.

The current investigation confirms that a short period of time (less than one week) off training can have positive effects on maximal strength expression in resistance trained athletes. It may also indicate that such changes are due to more efficient functioning of a less stressed neuromuscular system. Wider research of the strength training taper is also lacking (24), such research should look to determine how altering the volume and intensity of strength training may affect the effectiveness of tapering in strength athletes.

\section{PRACTICAL APPLICATIONS}

Based on the current findings it is clear that short term training cessation could be used by strength and conditioning practitioners as an effective means of tapering to enhance expression of maximal strength. It is suggested that athletes take a minimum of two days, but no more than a week, off from training prior to an important event where maximal strength expression may be beneficial, with around four days appearing to be optimal. Strength and conditioning practitioners should also consider the athletes previous training, as harder training periods may warrant longer periods of training cessation prior to events.

The findings also show that strength and conditioning coaches can design programs for their athletes where they may take short periods of time away from resistance training and be confident that athletes will maintain their previous levels of strength. Such a strategy could be applied as a planned recovery period in a training cycle, or when an athlete may have limited access to adequate training facilities. 


\section{REFERENCES}

1. Anderson T and Cattanach D. Effects of three different rest periods on expression of developed strength. Journal of Strength \& Conditioning Research 7: 185, 1993.

2. Brzycki M. Strength testing-predicting a one-rep max from reps-to-fatigue. Journal of Physical Education, Recreation \& Dance 64: 88-90, 1993.

3. Cormack SJ, Newton RU, and McGuigan MR. Neuromuscular and endocrine responses of elite players to an Australian rules football match. International Journal of Sports Physiology and Performance 3: 359-374, 2008.

4. Deschenes MR, Giles JA, McCoy RW, Volek JS, Gomez AL, and Kraemer WJ. Neural factors account for strength decrements observed after short-term muscle unloading. American Journal of Physiology Regulatory, Integrative and Comparative Physiology 282: R578-R583, 2002.

5. Ebben W, Feldmann C, Dayne A, Mitsche D, Alexander P, and Knetzger K. Muscle activation during lower body resistance training. International Journal of Sports Medicine 30: 1-8, 2009.

6. Escamilla RF, Francisco AC, Kayes AV, Speer KP, and Moorman 3rd C. An electromyographic analysis of sumo and conventional style deadlifts. Medicine and Science in Sports and Exercise 34: 682-688, 2002.

7. Folland JP and Williams AG. Morphological and neurological contributions to increased strength. Sports Medicine 37: 145-168, 2007.

8. Haff GG and Triplett NT. Essentials of Strength Training and Conditioning (4th Edition). Human Kinetics, 2015.

9. Hermens HJ, Freriks B, Disselhorst-Klug C, and Rau G. Development of recommendations for SEMG sensors and sensor placement procedures. Journal of Electromyography and Kinesiology 10: 361-374, 2000.

10. Hooper SL, Mackinnon LT, and Hanrahan S. Mood states as an indication of staleness and recovery. International Journal of Sport Psychology, 1997.

11. Hopkins WG. A scale of magnitudes for effect statistics. A New View of Statistics, 2002.

12. Hortobagyi T, Houmard JA, Stevenson JR, Fraser DD, Johns RA, and Israel RG. The effects of detraining on power athletes. Medicine and Science in Sports and Exercise 25: 929-935, 1993.

13. Hughes J, Chapman P, Brown S, Johnson N, and Stannard S. Indirect measures of substrate utilisation following exercise-induced muscle damage. European Journal of Sport Science 13: 509-517, 2013.

14. Izquierdo M, Ibanez J, Gonzalez-Badillo JJ, Ratamess NA, Kraemer WJ, Hakkinen K, Bonnabau H, Granados C, French DN, and Gorostiaga EM. Detraining and tapering effects on hormonal responses and strength performance. Journal of Strength \& Conditioning Research 21: 768-775, 2007.

15. Kilduff LP, Vidakovic P, Cooney G, Twycross-Lewis R, Amuna P, Parker M, Paul L, and Pitsiladis YP. Effects of creatine on isometric bench-press performance in resistance-trained humans. Medicine and Science in Sports and Exercise 34: 1176-1183, 2002.

16. Kraemer WJ and Ratamess NA. Hormonal responses and adaptations to resistance exercise and training. Sports Medicine 35: 339-361, 2005.

17. Linnamo V, Häkkinen K, and Komi P. Neuromuscular fatigue and recovery in maximal compared to explosive strength loading. European Journal of Applied Physiology and Occupational Physiology 77: 176-181, 1997.

18. Mangine G, Hoffman J, Fukuda D, Stout J, and Ratamess N. Improving muscle strength and size: The importance of training volume, intensity, and status. Kineziologija 47: 131-138, 2015.

19. McGuigan MR and Winchester JB. The relationship between isometric and dynamic strength in college football players. Journal of Sports Science \& Medicine 7: 101, 2008.

20. McGuigan MR, Winchester JB, and Erickson T. The importance of isometric maximum strength in college wrestlers. Journal of Sports Science \& Medicine 5: 108-113, 2006.

21. McLean BD, Coutts AJ, Kelly V, McGuigan MR, and Cormack SJ. Neuromuscular, endocrine, and perceptual fatigue responses during different length between-match microcycles in professional rugby league players. International Journal of Sports Physiology and Performance 5: 367-383, 2010.

22. Mujika I and Padilla S. Detraining: loss of training-induced physiological and performance adaptations. Part I. Sports Medicine 30: 79-87, 2000.

23. Pritchard H, Barnes M, Keogh J, and McGuigan M. The effectiveness of Prilepins chart for strength improvements in recreationally strength trained males, in: NSCA 2016 National Conference. New Orleans: National Strength and Conditioning Association, 2016.

24. Pritchard H, Keogh J, Barnes $\mathrm{M}$, and McGuigan M. Effects and mechanisms of tapering in maximizing muscular strength. Strength \& Conditioning Journal 37: 72-83, 2015. 
25. Pritchard HJ, Tod DA, Barnes MJ, Keogh JW, and McGuigan MR. Tapering practices of New Zealand's elite raw powerlifters. Journal of Strength \& Conditioning Research 30: 1796-1804, 2016.

26. Riad-Fahmy D, Read G, and Walker R. Salivary steroid assays for assessing variation in endocrine activity. Journal of Steroid Biochemistry 19: 265-272, 1983.

27. Rushall BS. A tool for measuring stress tolerance in elite athletes. Journal of Applied Sport Psychology 2: 51-66, 1990.

28. Storey AG, Birch NP, Fan V, and Smith HK. Stress responses to short-term intensified and reduced training in competitive weightlifters. Scandinavian Journal of Medicine \& Science in Sports 26: 29-40, 2015.

29. Twist $\mathrm{C}$ and Highton J. Monitoring fatigue and recovery in rugby league players. International Journal of Sports Physiology and Performance 8: 467-474, 2013.

30. Twist C, Waldron M, Highton J, Burt D, and Daniels M. Neuromuscular, biochemical and perceptual post-match fatigue in professional rugby league forwards and backs. Journal of Sports Sciences 30 : 359-367, 2012.

31. Weiss LW, Coney HD, and Clark FC. Optimal post-training abstinence for maximal strength expression. Research in Sports Medicine 11: 145-155, 2003.

32. Weiss LW, Wood LE, Fry AC, Kreider RB, Relyea GE, Bullen DB, and Grindstaff PD. Strength/power augmentation subsequent to short-term training abstinence. Journal of Strength \& Conditioning Research 18: 765-770, 2004.

\section{ACKNOWLEDGEMENTS}

We would like to thank those who volunteered to participate within the study.

\section{FIGURE LEGENDS}

Figure 1. Bodyweight Changes Over Time. Numbers represent testing times

Figure 2. Vastus Lateralis EMG Changes Over Time. Numbers represent testing times

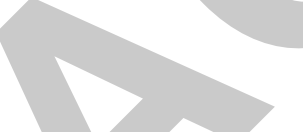

Figure 3. Tricep Brachii EMG Changes Over Time. Numbers represent testing times 


\begin{tabular}{|c|c|c|c|c|c|c|c|c|c|c|c|c|c|}
\hline \multirow[t]{2}{*}{ Day } & \multirow[t]{2}{*}{ Exercise } & \multicolumn{3}{|c|}{ Week One } & \multicolumn{3}{|c|}{ Week Two } & \multicolumn{3}{|c|}{ Week Three } & \multicolumn{3}{|c|}{ Week Four } \\
\hline & & Sets & Reps & $\%$ 1RM & Sets & Reps & $\%$ 1RM & Sets & Reps & $\%$ 1RM & Sets & Reps & $\% 1 R M$ \\
\hline 1 & Bench Press & 3 & 4 & 80 & 3 & 4 & 82.5 & 4 & 3 & 85 & 4 & 3 & 87.5 \\
\hline 1 & Back Squat & 4 & 6 & 75 & 4 & 6 & 77.5 & 4 & 4 & 80 & 4 & 4 & 82.5 \\
\hline 1 & Military Press & 4 & 6 & 75 & 4 & 6 & 77.5 & 4 & 4 & 80 & 4 & 4 & 82.5 \\
\hline 1 & Barbell Row & 3 & 10 & 70 & 3 & 10 & 72.5 & 4 & 8 & 75 & 4 & 8 & 77.5 \\
\hline 2 & Deadlift & 3 & 4 & 80 & 3 & 4 & 82.5 & 4 & 3 & $8 \overline{85}$ & 4 & 3 & 87.5 \\
\hline 2 & Close Grip Bench Press & 4 & 6 & 75 & 4 & 6 & 77.5 & 4 & 4 & 80 & 4 & 4 & 82.5 \\
\hline 2 & Deficit Deadlift (2") & 4 & 6 & 75 & 4 & 6 & 77.5 & 4 & 4 & 80 & 4 & 4 & 82.5 \\
\hline 2 & Good Morning & 3 & 10 & 70 & 3 & 10 & 72.5 & 4 & 8 & 75 & 4 & 8 & 77.5 \\
\hline 3 & Back Squat & 3 & 4 & 80 & 3 & 4 & 82.5 & 4 & 3 & 85 & 4 & 3 & 87.5 \\
\hline 3 & Paused Bench Press & 4 & 6 & 75 & 4 & 6 & 77.5 & 4 & 4 & 80 & 4 & 4 & 82.5 \\
\hline 3 & Front Squat & 4 & 6 & 75 & 4 & 6 & 77.5 & 4 & 4 & 80 & 4 & 4 & 82.5 \\
\hline 3 & Barbell Row & 3 & 10 & 70 & 3 & 10 & 72.5 & 4 & 8 & 75 & 4 & 8 & 77.5 \\
\hline
\end{tabular}

Table 1. Four Week Training Overview. (CG = Close Grip; 2ct Paused Bench = 2 second pause at the bottom of a bench press) 


\begin{tabular}{|c|c|c|c|c|c|c|}
\hline & \multicolumn{2}{|c|}{ Counter Movement Jump } & \multicolumn{2}{|c|}{ Mid-Thigh Pull } & \multicolumn{2}{|c|}{ Isometric Bench Press } \\
\hline Trial & $\begin{array}{l}\text { Jump Height } \\
(\mathrm{cm})\end{array}$ & $\begin{array}{c}\text { Flight Time : } \\
\text { Contraction Time }\end{array}$ & $\begin{array}{c}\text { Relative Peak } \\
\text { Force (N/kg) }\end{array}$ & $\operatorname{mRFD}\left(\mathbf{N} . \mathrm{s}^{-1}\right)$ & $\begin{array}{c}\text { Relative Peak } \\
\text { Force (N/kg) }\end{array}$ & $\operatorname{mRFD}\left(\mathbf{N} . \mathrm{s}^{-1}\right)$ \\
\hline A-1 & $37.7 \pm 6.0$ & $0.645 \pm 0.068$ & $37.3 \pm 3.3$ & $11248.4 \pm 3594.1$ & $18.5 \pm 2.7$ & $7801.3 \pm 2297.0$ \\
\hline A-2 & $37.7 \pm 5.3$ & $0.644 \pm 0.082$ & $38.3 \pm 5.3$ & $10206.4 \pm 2480.6^{*^{1}}$ & $19.5 \pm 2.3^{* 1}$ & $8533.9 \pm 3255.4^{*^{1}}$ \\
\hline A-3 & $39.4 \pm 5.3^{* 1,2}$ & $0.638 \pm 0.061$ & $39.1 \pm 5.1 *^{1}$ & $10386.0 \pm 3033.5^{*^{1}}$ & $19.8 \pm 2.7^{* 1}$ & $7850.6 \pm 2956.0$ \\
\hline B-1 & $37.3 \pm 3.2$ & $0.658 \pm 0.089$ & $37.7 \pm 6.6$ & $11758.1 \pm 2565.6$ & $18.8 \pm 2.8$ & $8418.9 \pm 2925.6$ \\
\hline B-2 & $39.2 \pm 3.6^{*^{1}}$ & $0.659 \pm 0.085$ & $38.8 \pm 5.8$ & $12061.6 \pm 4762.1$ & $19.6 \pm 3.1$ & $8627.2 \pm 4962.4$ \\
\hline B-3 & $39.8 \pm 5.4^{*^{1}}$ & $0.678 \pm 0.114$ & $39.7 \pm 6.5^{* 1}$ & $12811.0 \pm 3181.4^{*^{1}}$ & $19.8 \pm 2.9 *^{1}$ & $9449.5 \pm 3040.3^{*^{1}}$ \\
\hline
\end{tabular}

Table 2. Results of Performance Measures (mean \pm standard deviation). Letters represent the condition, $A=3.5$ days of training cessation condition; and, $B=5.5$ days of training cessation condition. Numbers represent testing time points, $1=$ pre-training; $2=$ on the final day of training, $3=$ following the respective training cessation period.

* indicates small ES vs time point of same condition, superscript number(s) indicates which time point. 


\begin{tabular}{|c|c|c|c|c|}
\hline Trial & $\begin{array}{c}\text { Salivary Cortisol } \\
(\mathbf{n g} / \mathbf{m l})\end{array}$ & $\begin{array}{c}\text { Salivary Testosterone } \\
(\mathbf{p g} / \mathbf{m l})\end{array}$ & $\begin{array}{c}\text { T/C Ratio } \\
(\mathbf{x 1 0 0 0})\end{array}$ & $\begin{array}{c}\text { Plasma Creatine } \\
\text { Kinase (I/U) }\end{array}$ \\
\hline A-1 & $5.90 \pm 2.07$ & $85.41 \pm 28.43$ & $16.53 \pm 8.84$ & $144.8 \pm 83.5$ \\
\hline A-2 & $5.38 \pm 2.01$ & $80.78 \pm 26.84$ & $16.21 \pm 6.60$ & $236.5 \pm 156.2$ \\
\hline A-3 & $4.62 \pm 1.12$ & $66.95 \pm 11.68$ & $14.84 \pm 2.38$ & $151.6 \pm 84.2$ \\
\hline B-1 & $5.71 \pm 1.84$ & $82.50 \pm 16.99$ & $15.38 \pm 3.90$ & $116.1 \pm 53.8$ \\
\hline B-2 & $5.41 \pm 1.43$ & $85.24 \pm 23.28$ & $17.81 \pm 10.07$ & $166.7 \pm 63.0$ \\
\hline B-3 & $4.83 \pm 0.97$ & $81.00 \pm 20.36$ & $17.53 \pm 5.89$ & $124.5 \pm 84.2$ \\
\hline
\end{tabular}

Table 3. Endocrine and Biochemical Measures. Letters represent the condition, $A=3.5$ days of training cessation condition; and, $B=5.5$ days of training cessation condition. Numbers represent testing time points, 1 = pre-training; 2 = on the final day of training, 3 = following the respective training cessation period. 


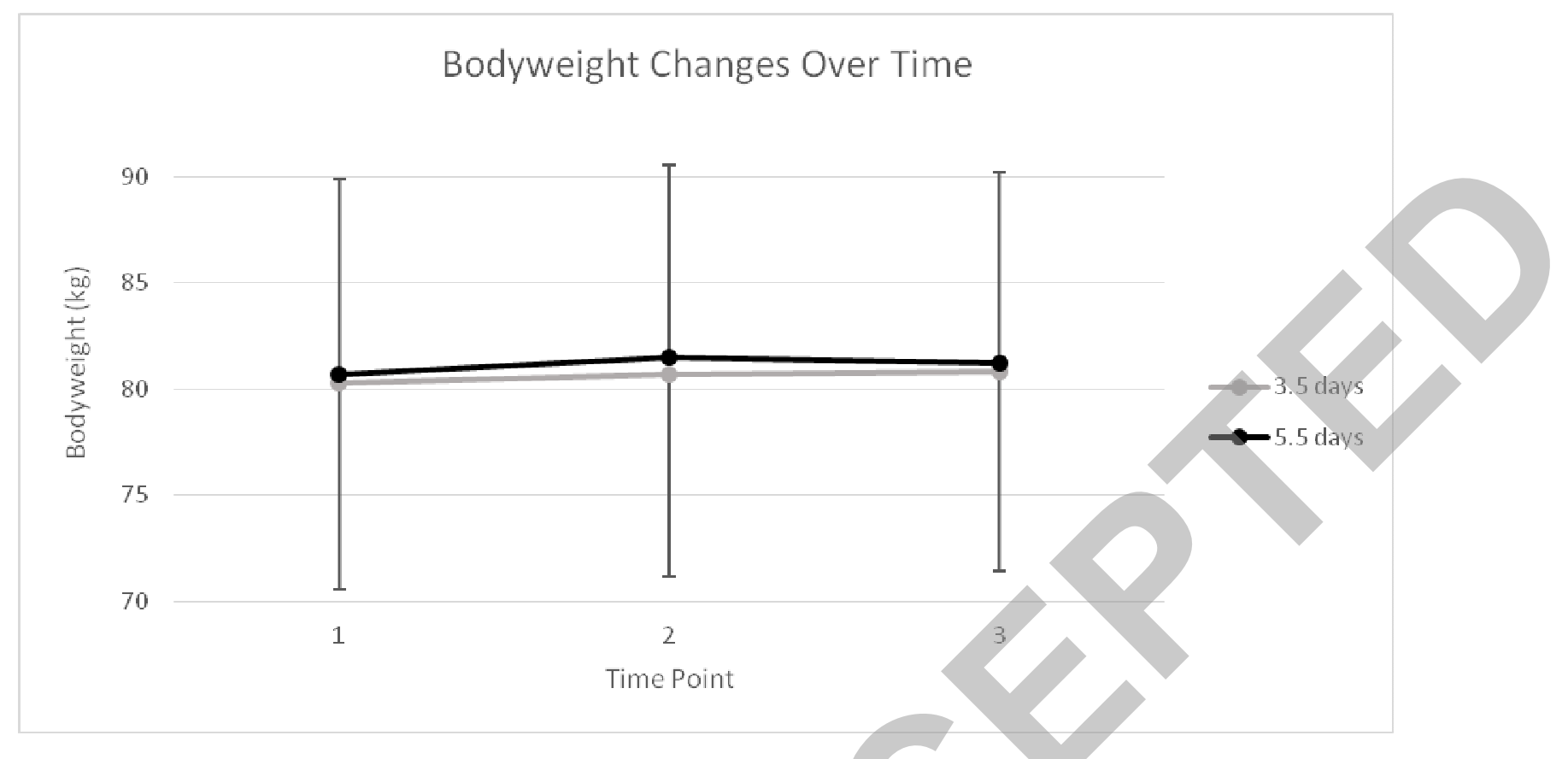

Figure 1. Bodyweight Changes Over Time. Numbers represent testing times, 1 = pre-training; 2 = on the final day of training, $3=$ following the respective training cessation period.

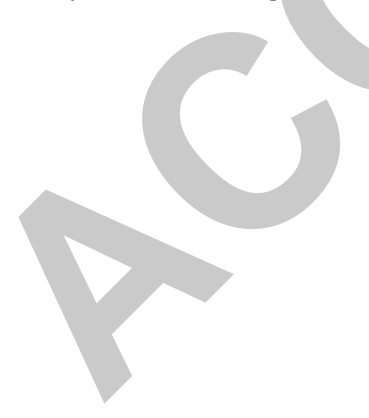




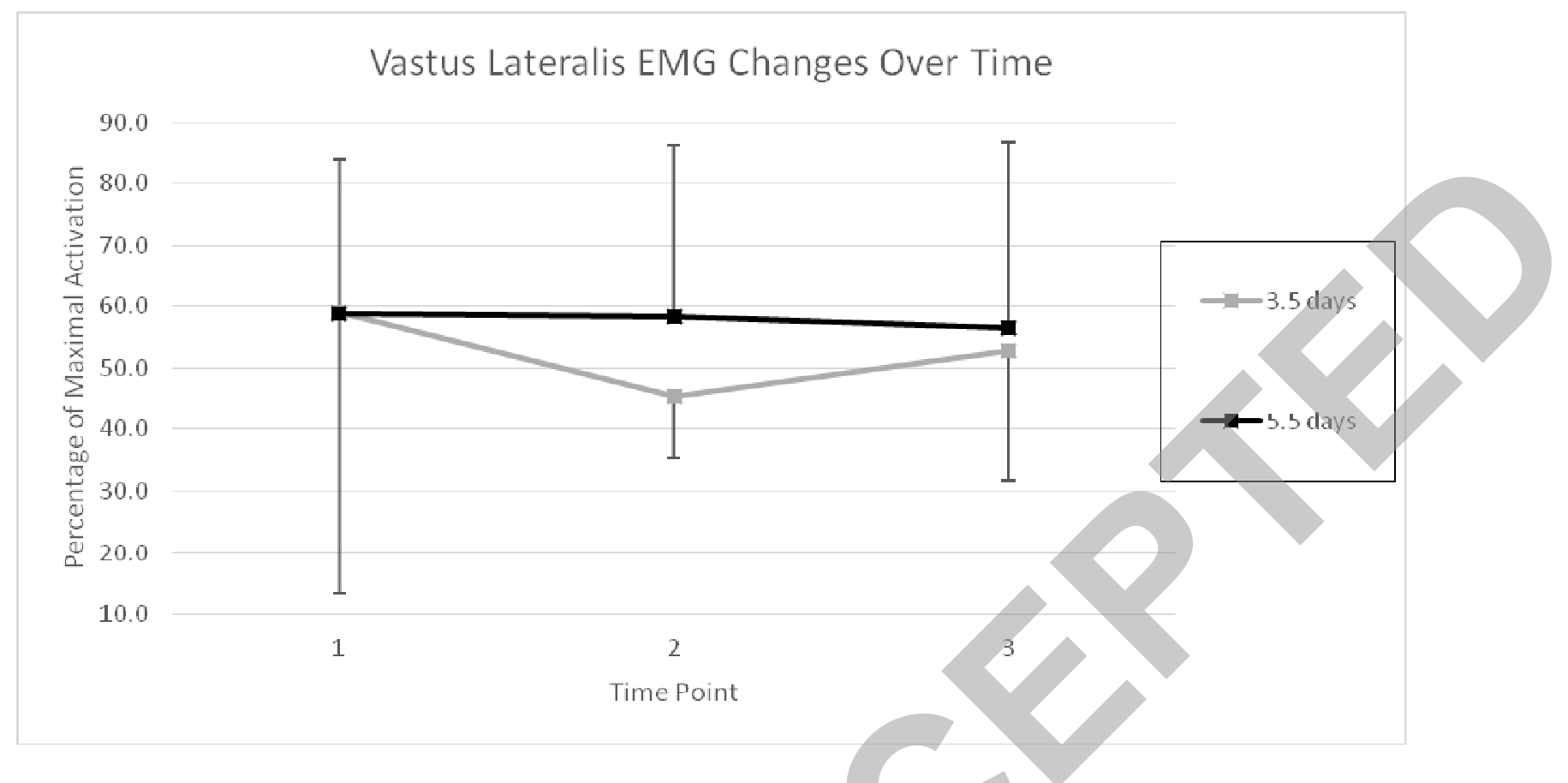

Figure 2. Vastus Lateralis EMG Changes Over Time. Numbers represent testing times, $1=$ pre-training; $2=$ on the final day of training, $3=$ following the respective training cessation period. 


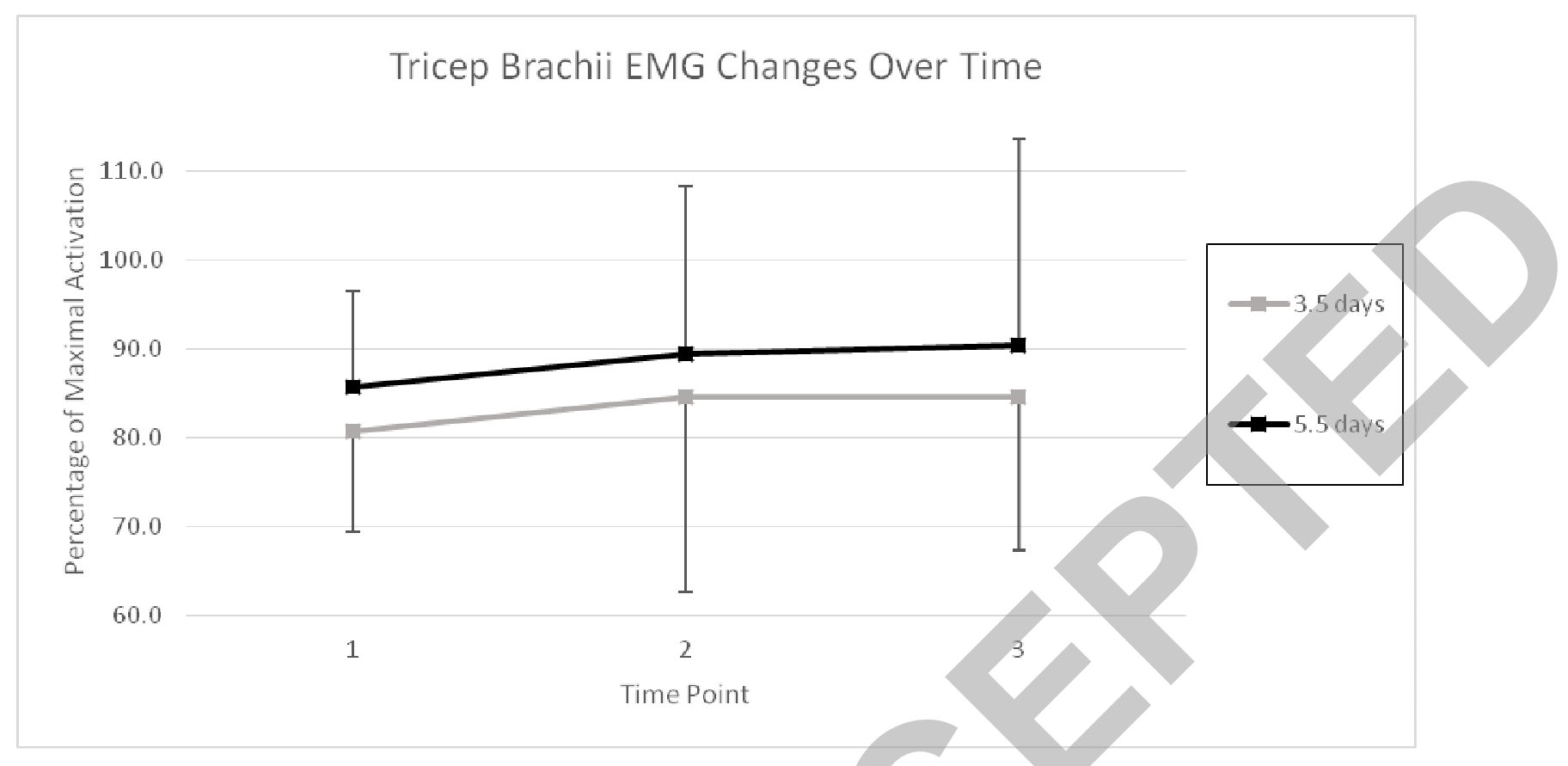

Figure 3. Tricep Brachii EMG Changes Over Time. Numbers represent testing times, $1=$ pre-training; 2 = on the final day of training, $3=$ following the respective training cessation period.

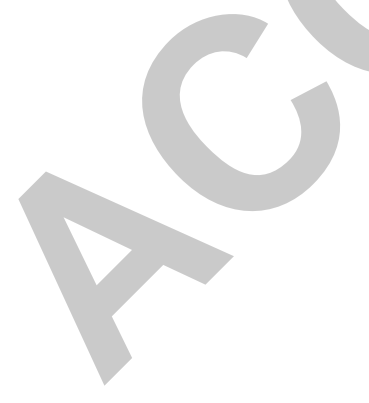

